\title{
Development of Functional Connectivity during Adolescence: A Longitudinal Study Using an Action-Observation Paradigm
}

\author{
Daniel J. Shaw ${ }^{1}$, Marie-Helene Grosbras ${ }^{2}$, Gabriel Leonard ${ }^{3}$, \\ G. Bruce Pike ${ }^{3}$, and Tomáš Paus ${ }^{1,3,4}$
}

\begin{abstract}
Successful interpersonal interactions rely on an ability to read the emotional states of others and to modulate one's own behavior in response. The actions of others serve as valuable social stimuli in this respect, offering the observer an insight into the actor's emotional state. Social cognition continues to mature throughout adolescence. Here we assess longitudinally the development of functional connectivity during early adolescence within
\end{abstract}

\section{INTRODUCTION}

The transition between childhood and adulthood involves numerous sociobehavioral and physiological developments. Among many behavioral features, adolescence is characterized by increases in sensation seeking and risk-taking (Steinberg, 2008), a peak in the greater display of physical aggression in men compared with women (Archer, 2004), and a shift from adult- to peer-directed social interactions (Spear, 2000). Such changes in social behavior likely both reflect and contribute toward developments in social cognition; if sensation seeking and risk-taking serve to elevate an individual's social standing, such proclivities might indicate the emergence of social awareness not dissimilar to that seen in adults. Similarly, social interactions with peers will contribute to the further maturation of an individual's social skills and the formation of social representations relevant for the autonomous life of a future adult (Spear, 2000). Adolescence, then, is a particularly interesting time to investigate the development of social cognition and its neural underpinnings.

Two networks of brain regions are believed to subserve social cognition in primates, and both demonstrate age-related changes in brain activity extending well into adolescence.

The first of these networks consists of regions that are engaged during the observation of someone performing an action. Some of these regions respond to action observation in the same way they do during the execu-

${ }^{1}$ University of Nottingham, ${ }^{2}$ University of Glasgow, ${ }^{3}$ McGill University, Montreal, Canada, ${ }^{4}$ University of Toronto two neural networks implicated in social cognition: one network of brain regions consistently engaged during action observation and another one associated with mentalizing. Using fMRI, we reveal a greater recruitment of the social-emotional network during the observation of angry hand actions in male relative to female adolescents. These findings are discussed in terms of known sex differences in adolescent social behavior. tion of that same or similar action. These "mirror-neuron regions"- so called because of their combined sensitivity to self and other action - were initially discovered in areas F5 and PF of the macaque brain (e.g., Fogassi, Gallese, Fadiga, \& Rizzolatti, 1998; Gallese, Fadiga, Fogassi, \& Rizzolatti, 1996; Rizzolatti, Fadiga, Fogassi, \& Gallese, 1996), and this functional property has led to suggestions that these brain regions underlie action understanding and/or imitation (Buccino, Binkofski, \& Riggioa, 2004; Rizzolatti, Fogassi, \& Gallese, 2001). In humans, neuroimaging investigations (e.g., Dinstein, Hasson, Rubin, \& Heeger, 2007) have since identified a similarly congruent response to both observed and executed actions within the premotor cortex (PMC), inferior frontal gyrus (IFG; the caudalmost section - pars opercularis - a likely homologue of F5), anterior inferior parietal lobule (IPL; the likely homologue of PF), and intraparietal sulcus (IPS). Passively observing another's actions also reliably engages posterior STS (for a review, see Allison, Puce, \& McCarthy, 2000), occipito-temporal cortex (MT/V5), and fusiform gyrus (Caspers, Zilles, Laird, \& Eickhoff, 2010). Using fMRI, we have described age-related decreases in brain activity within many nodes of this "action-observation network," including IFG, PMC, lateral temporal cortex, and fusiform gyrus, during early adolescence (Shaw, Grosbras, Leonard, Pike, \& Paus, 2011).

The second network believed to subserve social cognition is one consistently engaged during mentalizing tasks, whereby individuals are required to attribute mental states to others, such as intentions, desires, and beliefs. This network, termed by some authors the "social brain," 
includes medial pFC, ACC, posterior STS, temporal poles, TPJ, amygdala, anterior insula, and OFC (Pelphrey \& Carter, 2008a, 2008b; Frith, 2007; Frith \& Frith, 2007). The findings of developmental neuroimaging studies employing mentalizing tasks converge on an age-related decrease in brain activity between adolescence and adulthood within nodes of the social brain network (reviewed in Sebastian, Viding, Williams, \& Blakemore, 2010), again suggesting a protracted developmental course.

Like the maturation of social cognition, the development of brain activity within its putative neural substrates continues into adolescence. Importantly, there is considerable overlap between the brain regions comprising the action observation and social brain networks and those that continue to develop structurally throughout this developmental stage. Yet besides known structural changes in segregated regions of these networks, it is unclear how their integrated functioning emerges during adolescence. Developmental changes in brain activity will be especially evident within a network through which brain activity in one brain region is able to communicate with and modulate the activity in other interconnected regions, and both networks demonstrate considerable functional (e.g., Toro, Fox, \& Paus, 2008) and anatomical connectivity between their nodes. In terms of the action observation network, in the macaque brain, the STS is reciprocally connected with PF (Borra et al., 2008; Rizzolatti \& Matelli, 2003) that is, in turn, reciprocally connected with F5 (Rizzolatti \& Luppino, 2001). Within the social brain network, extensive reciprocal anatomical connections exist between the anterior insula, OFC, STS, and temporal pole (Augustine, 1996) and between the medial pFC and the STS (Barbas, Ghashghael, Dombrowski, \& Rempel-Clower, 1999; Bachevalier, Meunier, Lu, \& Ungerleider, 1997).

We set out to measure age-related changes in functional connectivity within the action observation and social brain networks using "seed" voxels representing, respectively, the network of brain regions engaged during the observation of hand actions (both angry and neutral) or specific to the processing of emotional hand actions (angry vs. neutral). Although the contrast used to define the latter network is expected to identify brain regions comprising the social brain, given the nature of the contrast we refer to it as the "socioemotional network" herein. On the basis of comparative neuroanatomical studies, we chose the IPS as our seed location for the action observation network for the following reasons. In the absence of direct visual input to frontal motor regions (Rizzolatti \& Luppino, 2001), STS is considered the main source of the visual input to the fronto-parietal circuit (e.g., Borra et al., 2008; Keysers \& Perrett, 2004), yet there are no direct anatomical connections between the STS and motor regions of the frontal cortex. Instead, the visuomotor transformations performed by subpopulations of neurons within IPS (Fogassi \& Luppino, 2005; Andersen, Snyder, Bradley, \& Xing, 1997; Rizzolatti, Fogassi, \& Gallese, 1997) make it a likely candidate for a relay between the STS and F5 (Keysers \& Perrett, 2004); within IPS, visual input could be converted into motor-compatible signals capable of modulating the activity of frontal-cortical motor circuits (see Goldman-Rakic, 1988, for a related discussion). Any changes in brain activity, then, should be reflected in changes in functional connectivity with this relay region. To measure developmental changes in brain function within the socioemotional network, we quantified functional connectivity between TPJ and the rest of the brain. This node of the network was selected on the basis of the abovementioned comparative anatomical connectivity studies, previous functional imaging studies demonstrating the consistent engagement of this region during mentalizing tasks (for a review, see Van Overwalle, 2010) and evidence of functional connectivity between this area and other nodes of the network in humans during the processing of socioemotional stimuli (Burnett \& Blakemore, 2009).

To explore functional connectivity, we draw on fMRI data collected longitudinally from a sample of young adolescents, scanned three times between 10 and 13 years using an action observation paradigm first described by Grosbras and Paus (2006). Specifically, to examine longitudinal changes in functional connectivity with the IPS, we measured covariation in BOLD signal between this region and the rest of the brain during the passive observation of hand actions relative to control nonbiological stimuli. To quantify developmental changes in functional connectivity with TPJ, we measured covariation between this region and the rest of the brain during passive observation of angry relative to emotionally neutral hand actions. The particular IPS and TPJ locations were defined as the corresponding areas pertaining to the circuit engaged during the respective task, as identified by a multivariate analysis. Changes in functional connectivity were quantified with a multivariate approach to seed voxel analysis, allowing us to measure age- and sex-related changes in patterns of covariance between the BOLD signal expressed by the seed voxel and the signal from the rest of the brain.

Using this dataset, we have reported previously on age-related changes in the BOLD signal measured in a set of brain regions defined by an activation-likelihood based meta-analysis of the action observation network (Shaw et al., 2011). In light of our previous findings, we expected to observe age-related changes in the degree of functional connectivity within these brain networks. Furthermore, given that sex differences in the expression of physical aggression peak during adolescence (Archer, 2004), we expected to see sex differences in the degree to which these networks are engaged by such socially evocative stimuli as angry hand movements.

\section{METHODS}

\section{Adolescents}

As part of an ongoing longitudinal study, a group of 65 adolescents (33 boys; 57 right-handers) were scanned at three 
time points. A detailed description of this sample is provided in Shaw et al. (2011). For reasons explained below, 19 adolescents ( 6 boys) were excluded from the present analyses. At Visit 1, the average age of the remaining sample was 10 years $(120.8$ months, $S D=4.7$ months; range $=$ 113-129 months), at Visit 2, the average age was 11.5 years (139 months, $S D=4.9$ months; range $=132-148$ months; mean time interval $=18.8$ months, $S D=0.9$ months), and at Visit 3, the average age was 13 years (157.8 months, $S D=$ 5.5 months; age range $=150-174$ months; time interval $=$ 18.9 months, $S D=2.3$ months). All adolescents reported English as their dominant language, and all had normal or corrected-to-normal vision. Informed consent was obtained from the parents, together with assent from the adolescents. The study conformed to the Helsinki declaration and was approved by the Research Ethics Board of the Montreal Neurological Institute (MNI).

\section{Stimuli}

The experiment involved the passive viewing of $2-5 \mathrm{sec}$ grayscale video clips depicting emotionally neutral and angry hand actions and control nonbiological motion stimuli. In each hand action clip, one of three actors was instructed to reach, grasp, and manipulate eight different objects (phone, pencil, spoon, computer mouse, glass, hammer, screwdriver, and cup) in line with their intended purpose, in either a neutral or angry manner. Each video clip started with the object alone; a left or right hand arriving from the right side of the screen then reached out, grasped, and manipulated the object, before disappearing back to where it had appeared. Only the hand and arm of the actor was visible.

The hand action stimuli employed in the present study were chosen from a sample of $\sim 200$ clips of angry, sad, and happy hand actions. A detailed description of the selection process is provided elsewhere (Grosbras \& Paus, 2006). In brief, for the angry hand actions, we selected 15 angry clips rated the highest on a 9-point scale for emotional intensity by four independent observers (mean = $7.23 \pm 0.50$ ); for the neutral clips, we chose 15 with the lowest score rating across all emotions (mean $=1.09 \pm$ 0.1 ). Importantly, the recognition of anger from the video clips was high across all the observers (84\%).

The selected video clips were arranged into ten 18-sec blocks, each block including four to seven video clips. In each block of video clips, $20 \%$ and $80 \%$ of the video clip duration contained, respectively, the arm movement alone and the hand-object interaction. There was no difference in this pattern between the angry and neutral clips; angry and neutral hand actions differed only in terms of the acceleration profile (for evidence that velocity of movement is a key kinematic element when discriminating angry from neutral hand actions, see Paterson, Pollick, \& Standford, 2001; Pollick, Paterson, Bruderlin, \& Sanford, 2001).

The control stimuli consisted of grayscale expanding and contracting concentric circles of various contrasts, roughly matching the contrast and motion characteristics of the hand action clips. These control stimuli were adapted from a study of Beauchamp, Lee, Haxby, and Martin (2003). A total of 10 blocks of control nonbiological motion were intermixed with 5 blocks of neutral and 5 blocks of angry hand actions. Two different orders were counterbalanced across subjects.

Stimuli were presented using Presentation (www. neurobehav.com), a software application permitting synchronization between stimuli presentation and MR image acquisition. Within the scanner, the stimuli were projected on a screen placed at the subjects' feet. Viewed through a mirror they subtended $10^{\circ} \times 7^{\circ}$ of visual angle. Examples of stimuli can be seen in Figure 1.

Adolescents were instructed to attend closely to the video clips and told that they would be asked questions about the stimuli after the scan. After scanning, we confirmed that the individual could recognize a subset of 10 hand action stimuli from a set of 14 clips (four oddballs).

\section{Imaging Protocol}

Scanning was performed on a 1.5T Siemens (Erlanger, Germany) Sonata scanner. A high-resolution T1-weighted structural image (matrix $=256 \times 256 \times 160 ; 1 \mathrm{~mm}^{3}$ voxels) was first acquired for anatomical localization and coregistration with the functional times series. The time series consisted of 180 T2*-weighted, gradient-echo, echoplanar BOLD images (matrix $=64 \times 64 \times 32 ; 4 \mathrm{~mm}^{3}$ voxels; $\mathrm{TR}=3 \mathrm{sec} ; \mathrm{TE}=50 \mathrm{msec}$ ) collected after the gradient had reached steady-state. Each slice was oriented parallel to a line connecting the base of the cerebellum to the base of OFC, covering the whole brain.

\section{Preprocessing}

Functional MRI data processing was carried out using FSL (fMRIB's Software Library, www.fmrib.ox.ac.uk/fsl). We excluded 19 adolescents (6 boys) due to severe image distortions caused by braces at one or more times; for the remaining 46 adolescents, we decided upon a lenient approach toward the removal of motion artifacts, as described next. Functional images were first corrected for head motion using MCFLIRT (Jenkinson, Bannister, Brady, \& Smith, 2002). We then identified visually timeseries that demonstrated residual motion artifacts after motion correction. For the 18 scans highlighted in this manner, we then used a second FSL tool ( $f s l_{\text {_motion }}$ outliers) to identify objectively specific affected volumes. These were defined as volumes containing changes in signal intensity that exceed a threshold based on outliers in the sum of squared changes. Affected volumes were omitted from subsequent analyses. This way we were able to include all remaining adolescents (46; 27 boys).

Affected volumes resulted from excessive head motion occurring between two successive volumes in the native time series (e.g., coughing, sneezing). We refer to this as 
Figure 1. Task and stimuli. Image depicting the task and stimuli employed in the present experiment from left to right: neutral hand action, angry hand action, control nonbiological motion. Neutral and angry hand actions differed only in terms of the acceleration profile. Modified from Grosbras and Paus (2006).

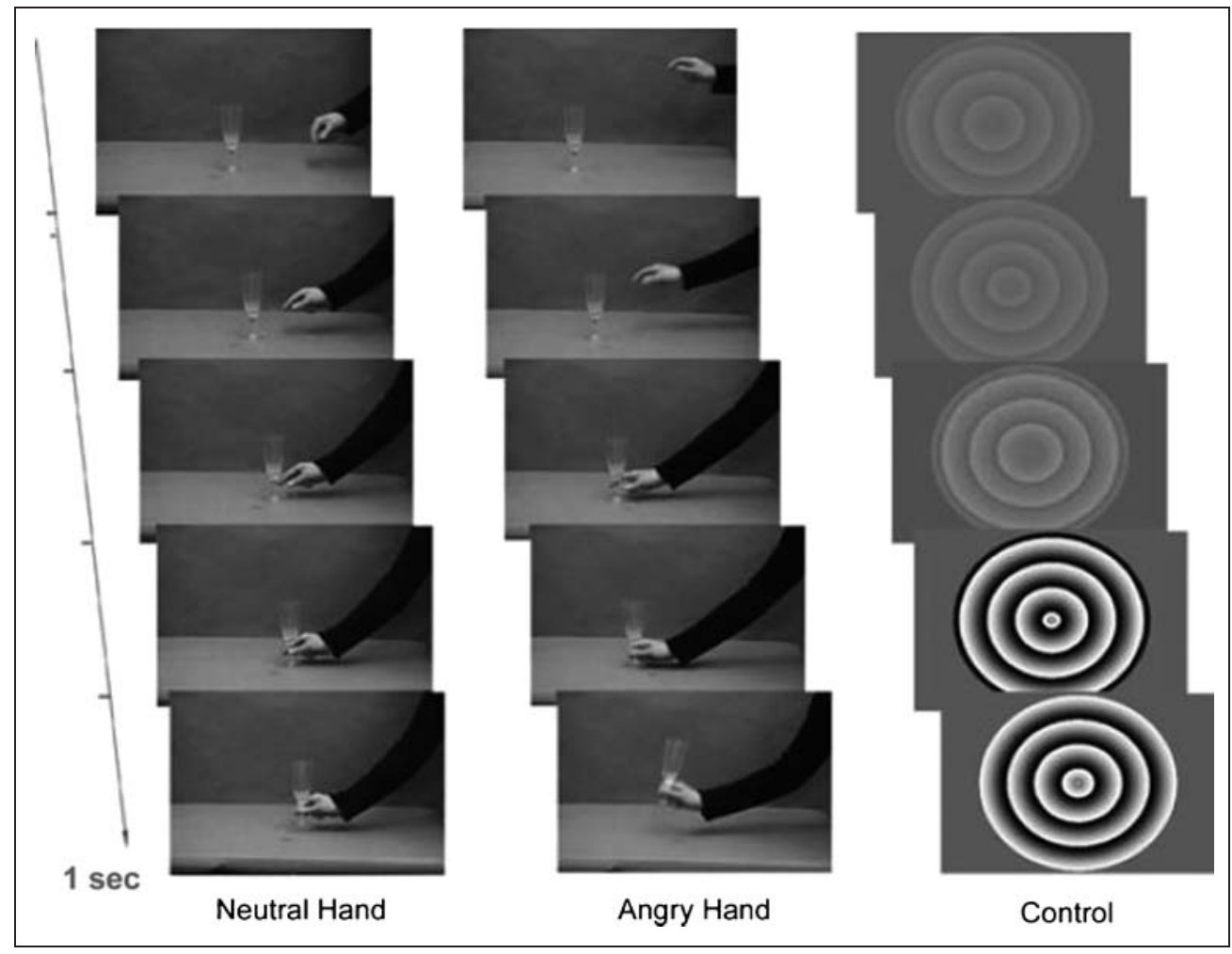

relative motion. The mean maximum relative motion for these 19 modified time series was $2.4 \mathrm{~mm}$ (range $=$ $0.8-6.0 \mathrm{~mm}$ ) compared with $0.5 \mathrm{~mm}$ (range $=0.2-$ $1.5 \mathrm{~mm}$ ) for the remaining unmodified scans.

Using FEAT v5.92, functional images were spatially smoothed using a 6.0-mm FWHM Gaussian kernel and high-pass filtered across time (Gaussian-weighted least squares straight line fitting; sigma $=50.0 \mathrm{sec}$ ). Time series were intensity normalized using grand mean scaling of the entire 4-D dataset by a single multiplicative factor to minimize unspecific time effects. Using FLIRT (Jenkinson \& Smith, 2001), all individual time series were then registered to the MNI-152 standard space template.

\section{Statistical Analyses: Partial Least Squares}

To examine age- and sex-dependent changes in functional connectivity within the action observation and socioemotional networks, we employed a multivariate approach to fMRI analysis - partial least squares (PLS). The first step was to localize seed voxels that best represent each network. To achieve this, we used two separate "Nonrotated Task PLS" analyses to contrast (1) the biological motion in the Angry and Neutral hand conditions with the nonbiological motion in the Control condition (i.e., Angry + Neutral vs. Control), and (2) the Angry condition with the Neutral condition (i.e., Angry vs. Neutral). With the seed voxels extracted from these two patterns of brain activity, we then set out to identify age and sex differences in the pattern of functional connectivity between these seed voxels and the rest of the brain. For this second step, we em- ployed two "Seed PLS" analyses. All analyses are described below.

PLS is a statistical technique that focuses on the relation between two or more "blocks" of variables and seeks a new set of variables that relate optimally the blocks using the fewest dimensions. Applied to neuroimaging data, PLS computes a "cross-block" matrix that represents the degree of covariance between a matrix containing a set of functional images and another containing a set of exogenous measures, such as the experimental protocol ("Task PLS") and/or the time series of one or more seed voxels ("Seed PLS"). Through singular value decomposition, a set of mutually orthogonal latent variable (LV) pairs is produced from the cross-block matrix. One element of the LV pair contains numerical weights for each task, creating a task profile that depicts common or task-related differences in covariance. The second identifies elements of the image that together exhibit the task profile and can be displayed in image space; this creates a "singular image" carrying the findings of the PLS, with numerical weights for each voxel representing the degree to which they express a given LV. The weights for both the task profile and the singular image are referred to as "saliences" (McIntosh, Chau, \& Protzner, 2004; McIntosh \& Lobaugh, 2004; McIntosh, Bookstein, Haxby, \& Grady, 1996). In a slight variation of this unconstrained, data-driven approach, contrasts between conditions can be defined a priori ("Nonrotated PLS"). This constrains the analysis to permit the identification of brain regions in which activity expresses a predicted task profile. 
To assess the statistical significance of LVs, permutation tests are used to estimate the probability of obtaining higher singular values to those obtained originally. The LV is considered significant if this probability is low $(p<.05)$ after 500 permutations (McIntosh \& Lobaugh, 2004; McIntosh et al., 2004). To determine the reliability of the contribution of each voxel to a given LV, the standard error for each salience in all LVs is estimated through bootstrap sampling. This involves varying the number of subjects within each condition. Unlike the permutation test, however, the assignment of subjects to conditions is maintained. As such, only the number of subjects contributing to task-related effects varies. Upon each bootstrap, new data matrices are created, and the PLS is recalculated. If the ratio of a salience to its standard error is greater than 2 (equivalent to a $z$ score of 2 if the distribution is Gaussian), the salience can be regarded as reliable; a salience that depends on which observations are included in the sample will be less precise than one that remains stable across different samples. Estimates of standard errors are usually stable after 100 bootstraps (McIntosh \& Lobaugh, 2004; McIntosh et al., 2004).

We performed PLS analyses within Matlab (Mathworks, Inc., Natick, MA; McIntosh \& Lobaugh, 2004). Performing two Nonrotated Task PLS analyses with 500 permutations and 200 bootstraps, we identified whole-brain patterns of brain activity that distinguished first between hand actions and nonbiological control motion and then between angry and emotionally neutral hand actions. Using voxels with the highest bootstrap ratio (BSR) value, within IPS and TPJ, from these Task PLS analyses as seed voxels, we ran separate Seed PLS analyses again using 500 permutations and 200 bootstraps to identify age-, sex-, and taskdependent patterns of functional connectivity between each seed voxel and the rest of the brain.

\section{RESULTS}

\section{Nonrotated Task PLS: Action Observation Effect}

To identify potential seed voxels for the subsequent IPS Seed PLS analysis, we first conducted a Nonrotational Task PLS analysis to contrast both angry and emotionally neutral hand actions with nonbiological motion (i.e., Angry + Neutral vs. Control). We included both boys and girls at all three visits in this analysis. The task profile and corresponding BSR image for this LV, herein referred to as the "action observation effect," are presented in Supplementary Figure 1. The action observation effect was significant $(p<.001)$. Table 1 reports the locations of peaks (BSR values) from this image; positive values are positively correlated to those conditions with positive weights in the corresponding task profile. As such, for this LV, voxels with positive values are engaged during the observation of biological (Angry and Neutral conditions) relative to nonbiological motion (Control condition).
Table 1. Coordinates of Peak Bootstrap Ratio Values for Action Observation Effect

\begin{tabular}{|c|c|c|c|c|c|c|}
\hline Index & Label & $x$ & $y$ & $z$ & $B S R$ & $\mathrm{~mm}^{3}$ \\
\hline 1 & Right MT/V5 & 46 & -70 & 8 & 19.05 & 33,536 \\
\hline 2 & Left MT/V5 & -50 & -62 & 12 & 13.94 & 33,605 \\
\hline 3 & Right IPS & 34 & -46 & 60 & 13.40 & 11,648 \\
\hline 4 & Right dorsal PMC & 22 & -14 & 52 & 11.17 & 4,480 \\
\hline 5 & Left IFG & -34 & 6 & 32 & 9.54 & 2,688 \\
\hline 6 & Left temporal pole & -38 & -2 & -40 & 9.05 & 640 \\
\hline 7 & Right SMG/AG & 54 & -30 & 32 & 8.88 & 4,672 \\
\hline 8 & Left cerebellum & -14 & -74 & -40 & 8.25 & 640 \\
\hline 9 & Left STS & -50 & -26 & -4 & 7.70 & 1,024 \\
\hline 10 & Left temporal pole & -50 & 14 & -24 & 7.66 & 12,800 \\
\hline 11 & Right cerebellum & 10 & -78 & -40 & 7.50 & 2,496 \\
\hline 12 & Left MFG & -38 & 18 & 24 & 7.40 & 2,688 \\
\hline 13 & Left amygdala & 26 & -6 & -12 & 6.44 & 576 \\
\hline 14 & Right PMC & 50 & 6 & -20 & 6.29 & 1,536 \\
\hline 15 & Right amygdala & 22 & -6 & -12 & 6.03 & 320 \\
\hline 16 & Left MOG & -18 & -90 & 16 & -11.62 & 17,472 \\
\hline 17 & Left STS & -62 & -14 & 8 & -8.32 & 3,136 \\
\hline 18 & Right ACC & 2 & 38 & 8 & -6.72 & 2,944 \\
\hline 19 & Right insula & 38 & -22 & 12 & -6.47 & 640 \\
\hline 20 & Left thalamus & -6 & -2 & 0 & -6.34 & 448 \\
\hline 21 & Left ACC & -6 & 22 & 28 & -6.28 & 704 \\
\hline 22 & Right fusiform gyrus & -14 & -64 & -8 & -6.05 & 384 \\
\hline
\end{tabular}

MNI-152 coordinates of voxels expressing peak BSR values in the corresponding BSR image for the action observation effect. Threshold = \pm 6 ; min. cluster extent $=5$ voxels. Positive BSR values are positively correlated to conditions with positive task saliences in the corresponding task profile; negative BSR values are positively correlated to conditions with negative task saliences. Abbreviations: SMG = supramarginal gyrus, $\mathrm{AG}=$ angular gyrus, $\mathrm{MFG}=$ middle frontal gyrus, $\mathrm{MOG}=$ middle occipital gyrus.

For reasons outlined above, we decided to use the voxel within the right IPS $(x=34, y=-46, z=60$; BSR value $=$ 13.40; indicated in bold in Table 1) as our seed voxel for the corresponding IPS Seed PLS analysis.

\section{Nonrotated Task PLS: Emotion Effect}

Again using a Nonrotated Task PLS, we next investigated whether a collection of brain regions existed throughout which BOLD signal was able to distinguish between angry and neutral hand actions. To achieve this, we contrasted the Angry with the Neutral condition (i.e., Angry vs. Neutral), including boys and girls at all three visits in the analysis. The task profile and corresponding BSR figure for 
Table 2. Coordinates of Peak Bootstrap Ratio Values for Emotion Effect

\begin{tabular}{|c|c|c|c|c|c|c|}
\hline Index & Label & $x$ & $y$ & $z$ & $B S R$ & $m m^{3}$ \\
\hline 1 & Left MOG & -18 & -86 & 28 & 5.38 & 23,360 \\
\hline 2 & Left insula & -42 & -6 & -8 & 5.05 & 4,992 \\
\hline 3 & Left SMA & -2 & 2 & 56 & 4.83 & 5,888 \\
\hline 4 & Left MTG & -66 & -18 & -4 & 4.62 & 1,216 \\
\hline 5 & Right TPJ & 62 & -30 & 24 & 4.60 & 4,224 \\
\hline 6 & Left TPJ & -58 & -30 & 20 & 4.55 & 6,272 \\
\hline 7 & Right PPC & 34 & -50 & 60 & 4.25 & 2,176 \\
\hline 8 & Left dorsal PMC & -46 & -2 & 44 & 4.16 & 960 \\
\hline 9 & Left PCC & -14 & -30 & 36 & 4.13 & 1,152 \\
\hline 10 & $\begin{array}{l}\text { Left dorsal } \\
\text { precentral gyrus }\end{array}$ & -10 & -14 & 76 & 4.04 & 768 \\
\hline 11 & Left STG & -50 & -26 & 8 & 3.40 & 1,216 \\
\hline 12 & Left OFC & -42 & 34 & 0 & 3.97 & 768 \\
\hline 13 & Left IPS & -34 & -42 & 60 & 3.59 & 960 \\
\hline 14 & Left cerebellum & -26 & -38 & -40 & 3.55 & 640 \\
\hline 15 & Right dorsal PMC & 46 & -10 & 52 & 3.38 & 640 \\
\hline 16 & Left MOG & -30 & -94 & 4 & -6.06 & 3,968 \\
\hline 17 & Right fusiform gyrus & 34 & -38 & -16 & -5.63 & 1,984 \\
\hline 18 & Right IOG & 34 & -86 & -8 & -4.22 & 2,240 \\
\hline 19 & Left fusiform gyrus & -34 & -50 & -20 & -3.78 & 1,280 \\
\hline
\end{tabular}

MNI-152 coordinates of voxels expressing peak BSR values in the corresponding BSR image for the emotion effect. Threshold $= \pm 3.0$; min. cluster extent $=10$ voxels. Positive BSR values are positively correlated to conditions with positive task saliences in the corresponding task profile; negative BSR values are positively correlated to conditions with negative task saliences. Abbreviations: $M O G=$ middle occipital gyrus, MTG $=$ middle temporal gyrus, STG $=$ superior temporal gyrus, $\mathrm{IOG}=$ inferior occipital gyrus.

this LV, herein referred to as the "emotion effect," are presented in Supplementary Figure 2. The emotion effect was significant $(p<.01)$. Table 2 reports the locations of peaks (BSR values) from this image. For this LV, voxels with positive values are engaged during the observation of angry relative to neutral hand actions. We selected the peak within the right TPJ region as our seed voxel in the corresponding Seed PLS analysis $(x=62, y=-30, z=$ $24, \mathrm{BSR}$ value $=4.60$; indicated in bold in Table 2 )

\section{Seed PLS: IPS}

Next, we conducted a Seed PLS to identify whether functional connectivity between the right IPS seed voxel and the rest of the brain differed between the Angry and Neutral conditions, and as a function of visit or sex. To do so, we included boys and girls at all three visits in the analysis.
The only significant $(p<.001)$ LV explained $44.39 \%$ of the cross-block variance. As shown in Figure 2, this LV reveals a task profile in which functional connectivity differs between the Angry and Neutral conditions only for boys at Visit 3. Table 3 presents the locations of peak BSR values.

To assess the significance of the Sex $\times$ Condition effect at Visit 3, we ran the above seed PLS across both boys and girls at this visit only. The only significant $(p<$ .001) LV to emerge confirms a Sex $\times$ Condition interaction explaining $65.51 \%$ of the cross-block variance. The task profile and corresponding BSR image are presented in Figure 3, and Table 4 presents the locations of peak BSR values. To aid interpretation of the Sex $\times$ Condition interaction at Visit 3, Supplementary Figure 3 plots the pairwise correlations between the right IPS seed voxel and six of the peaks presented in Table 4 . These plots suggest that the difference between men and women lies in the degree to which the network identified by the IPS Seed PLS-herein referred to the "action observation network"-is engaged under each condition, rather than the strength of the relationship between each nodes and the seed voxel. Specifically, the degree of BOLD response-at Visit 3- within these six nodes of the action observation network is lower under the Angry relative to the Neutral condition and more consistently so in boys compared with girls.

\section{Seed PLS: TPJ}

Next, we conducted a Seed PLS analysis to identify whether functional connectivity between the right TPJ seed voxel and the rest of the brain differentiated between the Angry and Neutral conditions, including boys and girls at all three visits in the analysis. The only significant $(p<$ .001) LV explained $37.95 \%$ of the cross-block variance. As shown in Figure 4, it reveals a task profile in which functional connectivity with the seed voxel differentiates between the Angry and Neutral condition at all visits in boys but only at Visit 1 in girls. The peak BSR values from this LV are presented in Table 5.

Finally, in light of the Sex $\times$ Condition effect at Visit 3 in the action observation network, we explored whether a Sex $\times$ Condition interaction existed at this visit within the socioemotional network. To do so, we ran the above TPJ Seed PLS across both boys and girls at Visit 3. The only significant $(p<.001)$ LV to emerge explains $53.50 \%$ of the cross-block variance. The task profile and corresponding BSR image are presented in Figure 5. These results suggest that, at Visit 3, functional connectivity between the right TPJ and the regions listed in Table 6 are roughly equivalent under both the Angry and Neutral condition in girls but is greater under the Angry relative to the Neutral condition in boys. To assess the accuracy of this interpretation, Supplementary Figure 4 plots the pairwise correlations between the right TPJ seed voxel and six of the peaks presented in Table 6 . This appears to confirm that, at Visit 3, activity throughout the socioemotional 
Figure 2. Task profile and BSR image for IPS Seed PLS for boys and girls across all visits. Top: Task profile for LV1 from IPS Seed PLS for both boys and girls (left and right of image, respectively) across all three visits. Conditions with positive weights are positively correlated with positive voxel saliences in the corresponding BSR image. This indicates that functional connectivity with the right IPS seed voxel differs between boys and girls only at Visit 3 . Bottom: Bootstrap ratio image thresholded at -6 to +6.5 ; radiological orientation (right $=$ left). Orange voxels contain positive saliences correlated positively with positive task weights in the corresponding task profile.

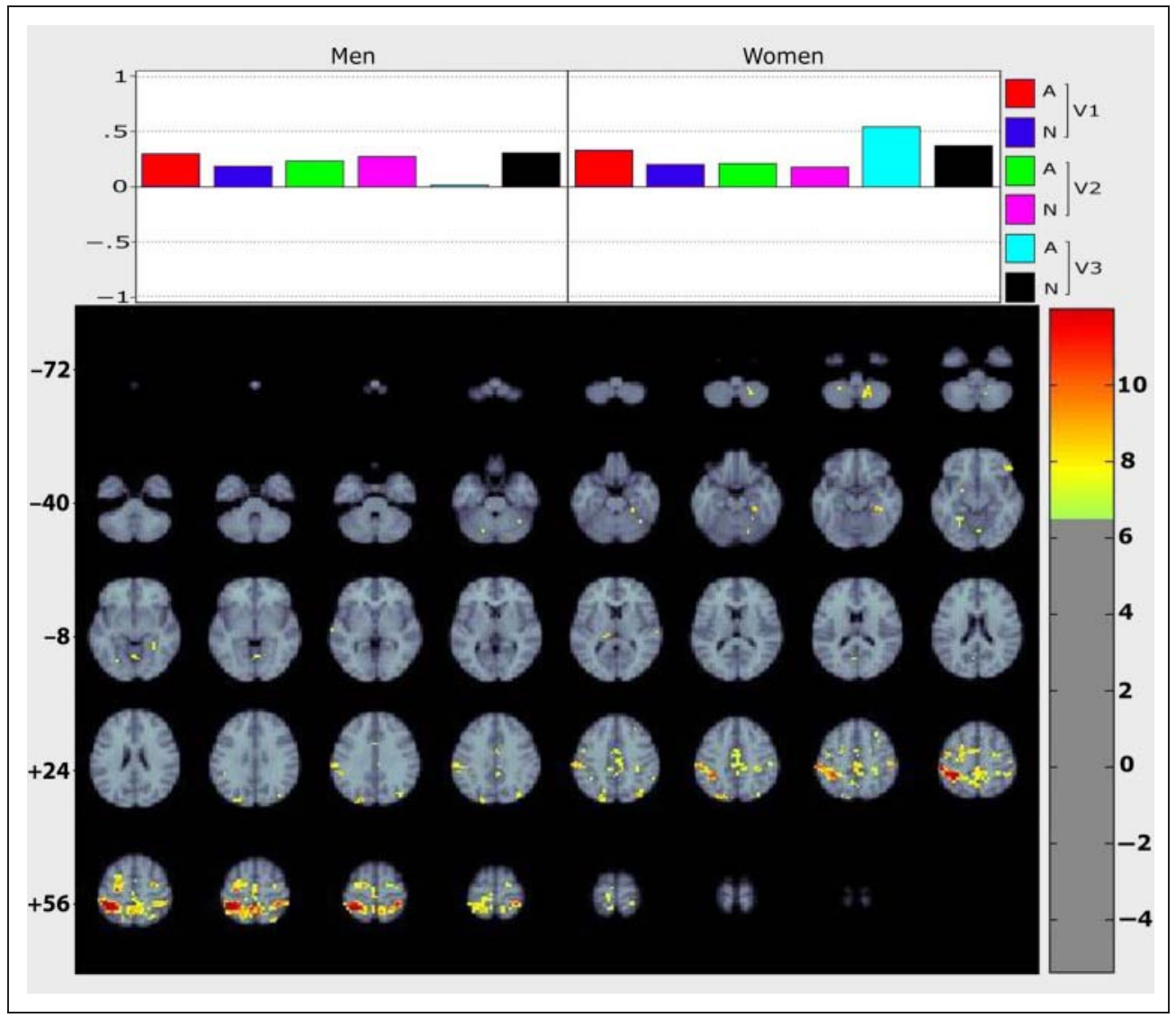

network is greater under the Angry relative to the Neutral conditions in boys but is equivalent across both conditions more than girls.

\section{DISCUSSION}

The current multivariate analyses of a longitudinal fMRI dataset obtained during the observation of angry and emotionally neutral hand actions revealed age and sex effects within the action observation and socioemotional brain networks during early adolescence. These results were generated in two steps: First, we identified the nodes of both networks by performing Nonrotated Task PLS analyses to reveal brain regions that exhibit, respectively, activity distinguishing biological (hand actions) from nonbiological motion, and those that differentiate between angry and emotionally neutral hand actions. Second, we carried out Seed PLS analyses to reveal three-way Age $\times$ Sex $\times$ Condition interactions in functional connectivity within the two networks.

The first Task PLS analysis contrasted both angry and emotionally neutral hand actions with control nonbiological motion. A fronto-parieto-temporal network expressed this action observation effect, including bilateral STS and fusiform gyrus, left IFG, and right PMC, IPL, and anterior IPS. This is consistent with fMRI studies examining the neural correlates of action observation (e.g., Grosbras \&
Paus, 2006; Buccino et al., 2001), revealing consistencies between the multivariate PLS approach to functional neuroimaging used here and the univariate general linear model approach used elsewhere.

Table 3. Coordinates of Peak Bootstrap Ratio Values for IPS Seed Voxel for Boys and Girls across All Visits

\begin{tabular}{llrrrrr}
\hline Index & \multicolumn{1}{c}{ Label } & $x$ & $y$ & $z$ & $B S R$ & $\mathrm{~mm}^{3}$ \\
\hline 1 & Right IPS & 34 & -46 & 60 & 489.08 & 30,976 \\
2 & Left IPL & -34 & -42 & 64 & 13.14 & 5,440 \\
3 & Right dorsal PMC & 26 & -18 & 56 & 11.47 & 4,736 \\
4 & Left dorsal PMC & -30 & -10 & 56 & 9.64 & 832 \\
5 & Right PPC/POJ & 22 & -78 & 44 & 9.35 & 1,728 \\
6 & Left ITG & -34 & -34 & -16 & 9.07 & 704 \\
7 & Left cerebellum & -22 & -54 & -48 & 8.34 & 1,088 \\
8 & Right postcentral gyrus & -54 & -26 & 48 & 8.52 & 640 \\
9 & Left PPC/POJ & -34 & -78 & 40 & 8.50 & 1,152 \\
\hline
\end{tabular}

MNI-152 coordinates of voxels expressing peak BSR values in the corresponding BSR image for the IPS Seed PLS for boys and girls across all visits. Threshold $=-5.5$ to +6.5 ; min. cluster extent $=10$ voxels. Positive BSR values are positively correlated to conditions with positive task saliences in the corresponding task profile; negative BSR values are positively correlated to conditions with negative task saliences. Abbreviations: $\mathrm{POJ}=$ parieto-occipital junction, ITG = inferior temporal gyrus. 
Figure 3. Task profile and BSR image for IPS Seed PLS for boys and girls at Visit 3 . Top: Task profile for LV1 from IPS Seed PLS for both boys and girls (left and right of image, respectively) at Visit 3 . Conditions with positive weights are positively correlated with positive voxel saliences in the corresponding BSR image. This indicates that functional connectivity with the right IPS seed voxel is no longer present under the Angry condition for boys at Visit 3 . Bottom: Bootstrap ratio image thresholded at -4.3 to +5.5 ; radiological orientation (right $=$ left). Orange voxels contain positive saliences correlated positively with positive task weights.

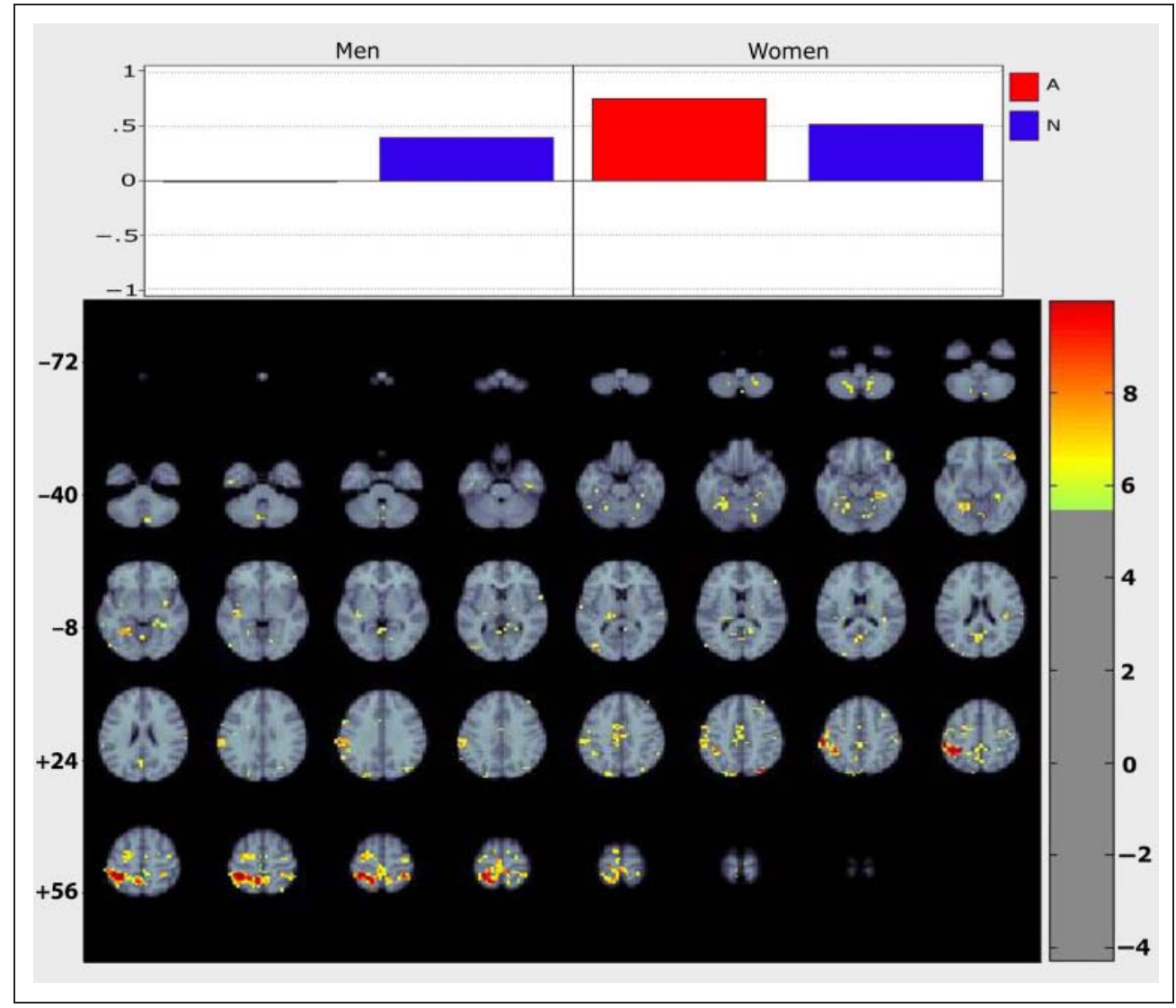

The second Task PLS analysis contrasted angry with neutral hand actions. An emotion effect was expressed by a network including TPJ, left OFC, IFG, insula, and temporal pole. Many of these brain regions are typically associated with nodes of the "social brain" (e.g., Frith, 2007; Frith \& Frith, 2007), with each node proposed to serve different aspects of high-level social cognition: TPJ is involved in processing others' complex actions, perhaps identifying the immediate underlying goal (see Van Overwalle, 2010, for a review), and the temporal poles are believed to combine complex perceptual inputs with an emotional response (Sebastian et al., 2010; Frith, 2007). Together this network is implicated in the ability to attribute mental states to others- that is, to mentalize. It is this ability that presumably allows one to be sensitive to the emotional connotation of the observed movements.

The next (Seed PLS) stage in the present analyses was to explore whether age-related changes exist in the pattern of functional connectivity within the action observation and socioemotional networks. We used the results of the above Task PLS analyses to define a seed voxel from each network. In light of anatomical connectivity, for the action observation network, this seed voxel was located within the right anterior IPS. At Visits 1 and 2, under the Angry and Neutral conditions, both boys and girls demonstrate a common level of activity within the action observation network. Specifically, activity within bilateral
Table 4. Coordinates of Peak Bootstrap Ratio Values for IPS Seed Voxel for Boys and Girls at Visit 3

\begin{tabular}{rlrrrrr}
\hline Index & \multicolumn{1}{c}{ Label } & $x$ & $y$ & $z$ & BSR & $m^{3}$ \\
\hline 1 & Right IPS & 34 & -46 & 60 & 2132.87 & 29,952 \\
2 & Right insula & 42 & -22 & -4 & 10.37 & 640 \\
3 & Left PPC/POJ & -30 & -78 & 44 & 9.53 & 896 \\
4 & Left IPS & -34 & -46 & 64 & 9.46 & 2,112 \\
5 & Right MOG & 38 & -82 & 8 & 9.39 & 768 \\
6 & Left OFC & -46 & 38 & -12 & 9.03 & 960 \\
7 & Right dorsal PMC & 26 & -6 & 56 & 8.90 & 3,776 \\
8 & Right precuneus & 10 & -82 & 48 & 8.46 & 1,984 \\
9 & Right striate & 2 & -66 & 16 & 8.23 & 1,664 \\
10 & Right thalamus & 14 & -26 & 8 & 7.99 & 832 \\
11 & Right fusiform gyrus & 22 & -54 & -8 & 7.91 & 3,264 \\
12 & Left fusiform gyrus & -34 & -30 & -20 & 7.87 & 1,536 \\
13 & Right anterior cingulate & 6 & -2 & 40 & 7.56 & 832 \\
14 & Right cerebellum & 18 & -54 & -52 & 6.98 & 768 \\
\hline
\end{tabular}

MNI-152 coordinates of voxels expressing peak BSR values in the corresponding BSR image from the IPS Seed PLS, for men and women at Visit 3. Threshold $=-5.0$ to +6.5 ; min. cluster extent $=10$ voxels. Positive BSR values are positively correlated to conditions with positive task saliences in the corresponding task profile; negative BSR values are positively correlated to conditions with negative task saliences. Abbreviations: POJ = parieto-occipital junction, $\mathrm{MOG}=$ middle occipital gyrus. 


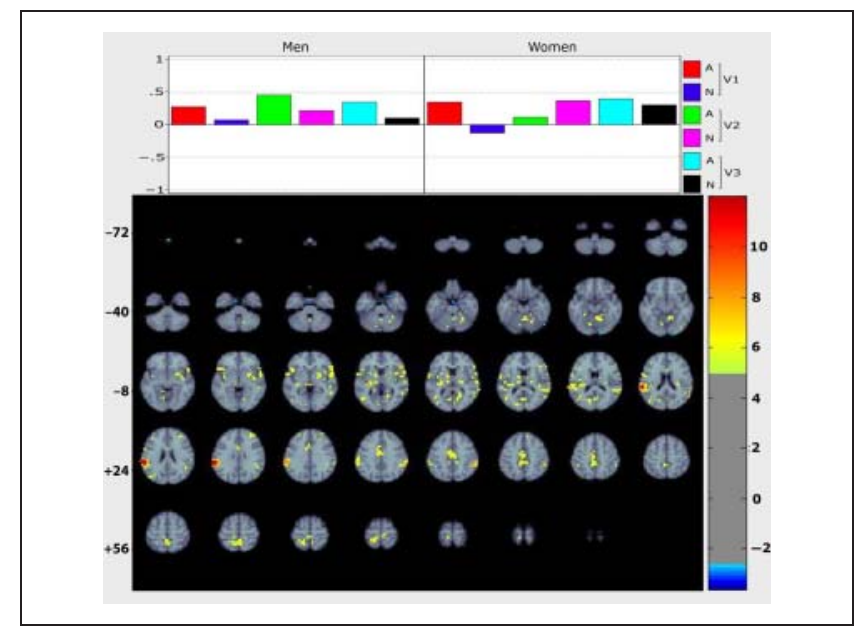

Figure 4. Task profile and BSR image for TPJ Seed PLS for boys and girls across all visits. Top: Task profile for LV1 for the TPJ Seed PLS for boys and girls left and right of image, respectively) across all visits. Conditions with positive weights are positively correlated with positive voxel saliences in the corresponding BSR image; conditions with negative weights are positively correlated with negative voxel saliences. This suggests that functional connectivity with the left TPJ seed voxel differentiates between the Angry and Neutral conditions, particularly at Visit 1 women and, to a lesser degree, at Visits 1 and 3 in boys. Bottom: Bootstrap ratio image thresholded at -3 to +5 ; radiological orientation (left $=$ right). Orange voxels contain positive saliences correlated positively with positive task weights in the corresponding task profile; blue voxels contain negative saliences that are positively correlated with negative task weights.

posterior parietal cortex (PPC) extending into the parietooccipital junction, fusiform gyrus and cerebellum, right IPL and dorsal PMC, and ipsilateral IPS and OFC covaries at a similar level of activity with the right IPS seed voxel across both conditions for both sexes at Visits 1 and 2. At Visit 3, however, boys do not show the same degree of activity within the action observation network under the Angry condition, whereas girls do so. This Sex $\times$ Condition interaction was confirmed by the subsequent IPS Seed PLS including data from Visit 3 only, which revealed the same Sex $\times$ Condition interaction. Pairwise correlations between the signal change in IPS seed voxel and the signal change in nodes of the network identified by the PLS analysis suggest that, at Visit 3, activity within the action observation network is lower under the Angry relative to the Neutral condition in boys.

In men at Visit 3, perhaps observing angry hand actions engages brain regions functionally distinct from the action observation network and involved in socioemotional processes. The findings of the second TPJ Seed PLS analysis lend some support to this proposition. In this analysis, we examined functional connectivity between the entire brain and a seed voxel in left TPJ—a region engaged during mentalizing tasks and processing social emotional stimuli. The pattern of functional connectivity with this TPJ seed voxel includes many of the nodes comprising the socioemotional network defined here, which itself closely resembles the "social brain" network (e.g., Frith, 2007; Frith \& Frith, 2007): bilateral temporal poles, TPJ, left OFC, and insula demonstrate functional connectivity with the seed voxel. Importantly, the corresponding task profile demonstrates that, in boys, this pattern of functional connectivity is more pronounced under the Angry relative to the Neutral condition, particularly at Visit 3 . The final TPJ Seed PLS and univariate pairwise correlations between parameter estimates of the contrast angry-neutral confirm that functional activity within the socioemotional network is greater under the Angry relative to the Neutral condition in men at Visit 3, whereas girls show equivalent degrees of activation throughout the network under both conditions.

If we take this evidence that men recruit the socioemotion network rather than the action observation network under the Angry condition at Visit 3, the fact that no functional connectivity is observed with the right IPS implies

Table 5. Coordinates of Peak Bootstrap Ratio Values for TPJ Seed Voxel for Boys and Girls across All Visits

\begin{tabular}{|c|c|c|c|c|c|c|}
\hline Index & Label & $x$ & $y$ & $z$ & $B S R$ & $m m^{3}$ \\
\hline 1 & Right TPJ & 62 & -34 & 24 & 1106.34 & 10,176 \\
\hline 2 & Left insula & 34 & -26 & 4 & 9.42 & 3,968 \\
\hline 3 & Left OFC & -54 & 26 & 4 & 8.33 & 4,928 \\
\hline 4 & $\begin{array}{l}\text { Right anterior } \\
\text { cingulate }\end{array}$ & 6 & -14 & 44 & 8.09 & 13,376 \\
\hline 5 & Left SMG/AG & -54 & -42 & 40 & 7.95 & 2,368 \\
\hline 6 & Left STS & -66 & -42 & 12 & 7.65 & 2,048 \\
\hline 7 & Right PMC & 58 & 6 & 4 & 7.34 & 2,304 \\
\hline 8 & Left insula & -42 & -6 & -8 & 7.27 & 832 \\
\hline 9 & Left putamen & -26 & 2 & -4 & 7.20 & 1,792 \\
\hline 10 & Left MT/V5 & 46 & -66 & 8 & 7.19 & 2,176 \\
\hline 11 & Left fusiform & -22 & -58 & -20 & 6.94 & 3,520 \\
\hline 12 & Right IFG & 46 & 18 & 0 & 6.74 & 1,152 \\
\hline 13 & Right STS & 66 & -22 & 4 & 6.41 & 768 \\
\hline 14 & Left prefrontal & -34 & 42 & 24 & 6.30 & 1,152 \\
\hline 15 & Right cerebellum & 10 & -70 & -16 & 6.24 & 832 \\
\hline 16 & Right striate & 2 & -70 & 12 & 6.24 & 1,216 \\
\hline 17 & Left thalamus & -22 & -18 & 8 & 6.01 & 640 \\
\hline 18 & Left lingual gyrus & -22 & -58 & 8 & 5.95 & 1,152 \\
\hline 19 & Left IOG & -14 & -98 & -4 & -3.60 & 768 \\
\hline
\end{tabular}

MNI-152 coordinates of voxels expressing peak BSR values in the corresponding BSR image from the TPJ Seed PLS for boys and girls across all visits. Threshold $=-3.5$ to +5.5 ; min. cluster extent $=10$ voxels. Positive BSR values are positively correlated to conditions with positive task saliences in the corresponding task profile; negative BSR values are positively correlated to conditions with negative task saliences. Abbreviation: $\mathrm{SMG}=$ supramarginal gyrus, $\mathrm{AG}=$ angular gyrus, IOG $=$ inferior occipital gyrus. 
Figure 5. Task profile and BSR image for TPJ Seed PLS for boys and girls at Visit 3. Top: Task profile for LV1 for the TPJ Seed PLS for boys and girls (left and right of image, respectively) at Visit 3 . Conditions with positive weights are positively correlated with positive voxel saliences in the corresponding BSR image. This suggests that functional connectivity with the left TPJ seed voxel is reduced under the Neutral condition for boys relative to girls. Bottom: Bootstrap ratio image thresholded at \pm 4 ; radiological orientation (left $=$ right). Orange voxels contain positive saliences correlated positively with positive task weights in the corresponding task profile.

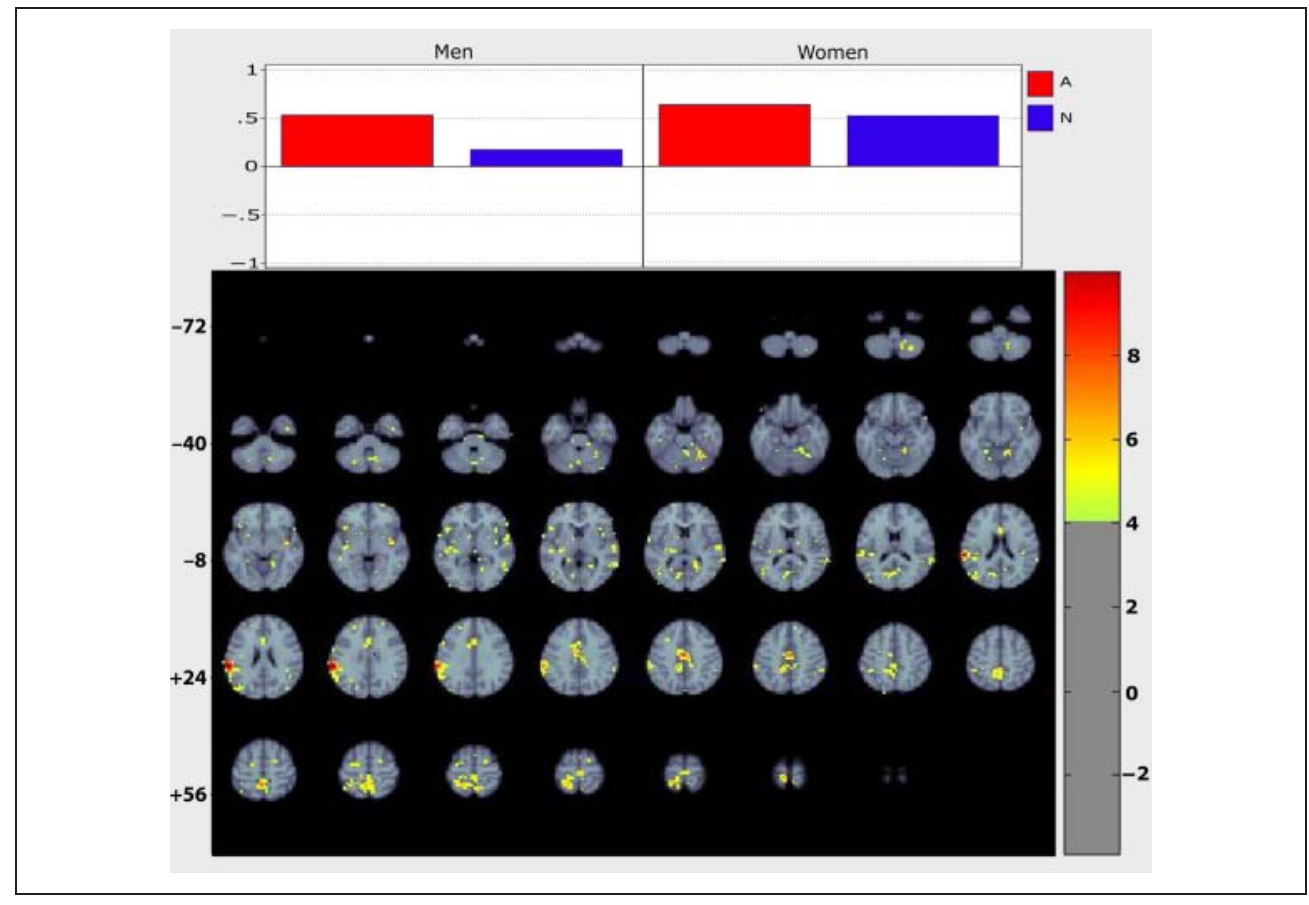

the "social brain" network takes its input from elsewhere. One possibility is that the action observation network provides the input to this higher-order network. The social and cognitive processes underlying the ability to mentalize on the basis of only observed actions will range from low-level perceptual processes involved in biological motion processing to those enabling us to perceive and understand others' emotional responses (Sebastian et al., 2010; Frith \& Frith, 2007). As such, considerable functional overlap will exist between the functions of the action observation and the socioemotional network, whereas the former network appears to be involved in simulating others' motor actions, the latter may be responsible for simulating others' (emotional) mental states (Uddin, Iacoboni, Lange, \& Keenan, 2007). Both networks seemingly contribute toward different aspects of social information processing. It is suggested that the low-level processing of observed actions performed within the action observation network might provide the input to the higher-level mentalizing network (for a review, see Van Overwalle, 2010; Van Overwalle \& Baetens, 2009). If this were the case, direct anatomical connections would exist between nodes of both networks. Indeed, in the monkey such connections exist between the posterior cingulate cortex, PPC, and IPL (e.g., Lou et al., 2004; Carmen \& Goldman-Rakic, 1989) and between anterior insula and OFC, PMC, STS, and temporal pole (Augustine, 1996). The present analyses revealed two regions common to both networks, namely the OFC and insula; based on their connectivity, these two regions could act as relays between the action observation network and the "social brain" permitting continuous communication between them.
Table 6. Coordinates of Peak Bootstrap Ratio Values for TPJ Seed Voxel for Boys and Girls at Visit 3

\begin{tabular}{|c|c|c|c|c|c|c|}
\hline Index & Label & $x$ & $y$ & $z$ & $B S R$ & $m m^{3}$ \\
\hline 1 & Right TPJ & 62 & -34 & 24 & 939.66 & 10,432 \\
\hline 2 & Right cingulate/SMA & 2 & -10 & 40 & 9.06 & 7,104 \\
\hline 3 & Left temporal pole & -30 & 10 & -24 & 7.67 & 2,752 \\
\hline 4 & Right precuneus & 2 & -50 & 56 & 7.45 & 14,976 \\
\hline 5 & Left insula & -38 & -6 & -8 & 7.33 & 832 \\
\hline 6 & Left cerebellum & -14 & -58 & -44 & 6.93 & 1,216 \\
\hline 7 & Right insula & 38 & 18 & -4 & 6.81 & 896 \\
\hline 8 & Left STS & -62 & -46 & 12 & 6.71 & 1,600 \\
\hline 9 & Right striate & 10 & -70 & 16 & 6.17 & 2,112 \\
\hline 10 & Right MOG & 42 & -78 & 20 & 5.99 & 1,536 \\
\hline 11 & Left SFG & -18 & -2 & 64 & 5.96 & 768 \\
\hline 12 & Right SFG & 22 & -6 & 60 & 5.88 & 1,024 \\
\hline 13 & Right insula & 34 & -26 & 8 & 5.83 & 768 \\
\hline 14 & Left STS & -62 & -14 & 4 & 5.54 & 896 \\
\hline 15 & Right STG & 46 & -26 & 0 & 5.43 & 896 \\
\hline
\end{tabular}

MNI-152 coordinates of voxels expressing peak BSR values in the corresponding BSR image from the TPJ Seed PLS for boys and girls at Visit 1. Threshold $= \pm 5$; min. cluster extent $=10$ voxels. Positive BSR values are positively correlated to conditions with positive task saliences in the corresponding task profile; negative BSR values are positively correlated to conditions with negative task saliences. Abbreviations: $\mathrm{MOG}=$ middle occipital gyrus, $\mathrm{SFG}=$ superior frontal gyrus, $\mathrm{STG}=$ superior temporal gyrus. 
Should the socioemotional network operate upon the output of the action observation network, we might also expect both networks to exhibit similar protracted development courses. Consistent with this proposition, nodes of both networks demonstrate age-related decreases in BOLD signal that continue throughout adolescence (Shaw et al., 2011; Sebastian et al., 2010). Parallel developmental trajectories in functional brain activity within nodes of both networks might be indicative of the interdependence between them.

The presence of a clear sex effect at Visit 3, whereby observing angry hand actions engages the socioemotional network instead of the action observation for boys is consistent with known sex differences in social behavior. Archer's (2004) meta-analysis revealed large sex effects in some displays of aggression, with men expressing more physical aggression than women. Importantly, this sex effect peaks during adolescence. A sex difference in the proclivity for physical aggression might be driven by sex differences in threat processing (McClure, 2000). Interestingly, in a large-scale study of face processing, we have shown that the amygdala is more engaged in male compared with female adolescents in response to angry faces, despite a stronger response in cortical regions (Tahmasebi et al., 2011). By revealing that the higher-level socioemotional network becomes more involved than the lowlevel action observation network in response to angry hand actions in boys but not girls, our findings lend further support to this proposition of a brain-behavior relationship. Further experiments are certainly needed to help establish such a link between brain functional connectivity and socioemotional abilities.

\section{Conclusions}

Developmental social neuroscience has tended to relate the emergence of abilities subsumed by social cognition to the neurodevelopment of specific nodes of brain networks, particularly nodes of the "social brain." Recently, however, it has been suggested that this focus should shift to the development of connectivity between these nodes (Pelphrey \& Carter, 2008a, 2008b). The findings of the present study further emphasize the importance of this latter approach; we demonstrate that a change in functional connectivity with the right IPS and TPJ during the observation of angry hand actions may reveal a shift in the brain network engaged during the observation of socially relevant cues. Moreover, our data suggest that sex differences are present in the two networks but at different stages of adolescence.

\section{Acknowledgments}

This work was funded by the Sante Fe Institute Consortium and the Canadian Institutes of Health Research. We thank Candice Cartier, Elissa Golden, Valerie Legge, Kristina Martinu, and Line Gingras for assistance with the recruitment of participants and data collection. We are grateful to the participating families for their long-term commitment and interest in this work.
Reprint requests should be sent to Tomáš Paus, Rotman Research Institute, 3560 Bathurst St., Toronto, Ontario, M6A 2EI, Canada, or via e-mail: tpaus@rotman-baycrest.on.ca.

\section{REFERENCES}

Allison, T., Puce, A., \& McCarthy, G. (2000). Social perception from visual cues: Role of the STS region. Trends in Cognitive Sciences, 4, 267-278.

Andersen, R. A., Snyder, L. H., Bradley, D. C., \& Xing, J. (1997). Multimodel representations of space in the posterior parietal cortex and its use in planning movements. Annual Review of Neuroscience, 20, 303-330.

Archer, J. (2004). Sex differences in aggression in real-world settings: A meta-analytic review. Review of General Psychology, 8, 291-322.

Augustine, J. R. (1996). Circuitry and functional aspects of the insula lobe in primates including humans. Brain Research Reviews, 22, 229-244.

Bachevalier, J., Meunier, M., Lu, M. X., \& Ungerleider, L. G. (1997). Thalamic and temporal cortex input to medial prefrontal cortex in rhesus monkeys. Experimental Brain Research, 115, 430-444.

Barbas, H., Ghashghael, S. M., Dombrowski, S. M., \& Rempel-Clower, N. L. (1999). Medial prefrontal cortices are unified by common connections with superior temporal cortices and distinguished by input from memory-related areas in the rhesus monkey. Journal of Comparative Neurology, 410, 343-367.

Beauchamp, M. S., Lee, K. E., Haxby, J. V., \& Martin, A. (2003). fMRI responses to video and point-light displays of moving humans and manipulable objects. Journal of Cognitive Neuroscience, 15, 991-1001.

Borra, E., Belmalih, A., Calzavara, R., Gerbella, M., Murata, A., Rozzi, S., et al. (2008). Cortical connections of macaque anterior intraparietal (AIP) area. Cerebral Cortex, 18, 1094-1111.

Buccino, G., Binkofski, F., Fink, G. R., Fadiga, L., Fogassi, L., Gallese, V., et al. (2001). Action observation activates premotor and parietal areas in a somatotopic manner: An fMRI study. European Journal of Neuroscience, 13, 400-404.

Buccino, G., Binkofski, F., \& Riggioa, L. (2004). The mirror neuron system and action recognition. Brain and Language, 89, 370-376.

Burnett, S., \& Blakemore, S.-J. (2009). Functional connectivity during a social emotional task in adolescences and in adults. European Journal of Neuroscience, 29, 1294-1301.

Carmen, C., \& Goldman-Rakic, P. S. (1989). Posterior parietal cortex in rhesus monkey: 1. Parcellation of areas based on distinctive limbic and sensory corticocortical connections. The Journal of Comparative Neurology, 287, 393-421.

Caspers, S., Zilles, K., Laird, A. R., \& Eickhoff, S. (2010). ALE meta-analysis of action observation and imitation in the human brain. Neuroimage, 50, 1148-1167.

Dinstein, I., Hasson, U., Rubin, N., \& Heeger, D. J. (2007). Brain areas selective for both observed and executed movements. Journal of Neurophysiology, 98, 1415-1427.

Fogassi, L., Gallese, V., Fadiga, L., \& Rizzolatti, G. (1998). Neurons responding to the sight of goal-directed hand/arm actions in the parietal area $\mathrm{PF}(7 \mathrm{~b})$ of the macaque monkey. Society of Neuroscience Abstracts, 24, 154.

Fogassi, L., \& Luppino, G. (2005). Motor functions of the parietal lobe. Current Opinion in Neurobiology, 15, 525-631.

Frith, C. D. (2007). The social brain? Philosophical

Transactions of the Royal Society of London, Series B, Biological Sciences, 362, 671-678. 
Frith, C. D., \& Frith, U. (2007). Social cognition in humans. Current Biology, 17, R724-R732.

Gallese, V., Fadiga, L., Fogassi, L., \& Rizzolatti, G. (1996). Action recognition in the premotor cortex. Brain, 119, 593-609.

Goldman-Rakic, P. S. (1988). Topography of cognition-Parallel distributed networks in primate association cortex. Annual Review of Neuroscience, 11, 137-156.

Grosbras, M., \& Paus, T. (2006). Brain networks involved in viewing angry hands or faces. Cerebral Cortex, 16, 1087-1096.

Jenkinson, M., Bannister, P., Brady, M., \& Smith, S. (2002). Improved optimisation for the robust and accurate linear registration and motion correction of brain images. Neuroimage, 17, 825-841.

Jenkinson, M., \& Smith, S. (2001). A global optimisation method for robust affine registration of brain images. Medical Image Analysis, 5, 143-156.

Keysers, C., \& Perrett, D. I. (2004). Demystifying social cognition: A Hebbian perspective. Trends in Cognitive Sciences, 8, 501-507.

Lou, H. C., Luber, B., Crupain, M., Keenan, J. P., Nowark, M., Kjaer, T. W., et al. (2004). Parietal cortex and representation of the mental self. Proceedings of the National Academy of Sciences, U.S.A., 101, 6827-6832.

McClure, E. B. (2000). A meta-analytic review of sex differences in facial expression processing and their development in infants, children, and adolescents. Psychological Bulletin, 126, 424-453.

McIntosh, A. R., Bookstein, F. L., Haxby, J. V., \& Grady, C. L. (1996). Spatial pattern analysis of functional brain images using partial least squares. Neuroimage, 3, 143-157.

McIntosh, A. R., Chau, W. K., \& Protzner, A. B. (2004). Spatiotemporal analysis of event-related fMRI data using partial least squares. Neuroimage, 23, 764-775.

McIntosh, A. R., \& Lobaugh, N. J. (2004). Partial least squares analysis of neuroimaging data: Applications and advances. Neuroimage, 23, S250-S263.

Paterson, H. M., Pollick, F. E., \& Standford, A. J. (2001). The role of velocity in affect discrimination. In Proceedings of the Twenty-Third Annual Conference of the Cognitive Science (pp. 756-761).

Pelphrey, K. A., \& Carter, E. J. (2008a). Charting the typical and atypical development of the social brain. Development and Psychopatbology, 20, 1081-1102.

Pelphrey, K. A., \& Carter, E. J. (2008b). Brain mechanisms for social perception; lessons from autism and typical development. Annals of the New York Academy of Sciences, $1145,283-299$.
Pollick, F. E., Paterson, H. M., Bruderlin, A., \& Sanford, A. J. (2001). Perceiving affect from arm movement. Cognition, 82, B51-B61.

Rizzolatti, G., Fadiga, L., Fogassi, L., \& Gallese, V. (1996). Premotor cortex and the recognition of motor actions. Cognitive Brain Research, 3, 131-141.

Rizzolatti, G., Fogassi, L., \& Gallese, V. (1997). Parietal cortex: From sight to action. Current Opinion in Neurobiology, 7, 562-567.

Rizzolatti, G., Fogassi, L., \& Gallese, V. (2001). Neurophysiological mechanisms underlying the understanding and imitation of action. Nature Reviews Neuroscience, 2, 661-670.

Rizzolatti, G., \& Luppino, G. (2001). The cortical motor system. Neuron, 31, 889-901.

Rizzolatti, G., \& Matelli, M. (2003). Two different streams form the dorsal visual stream: Anatomy and functions. Experimental Brain Research, 153, 146-157.

Sebastian, C., Viding, E., Williams, K. D., \& Blakemore, S.-J. (2010). Social brain development and the affective consequence of ostracism in adolescence. Brain and Cognition, 72, 134-145.

Shaw, D. J., Grosbras, M.-H., Leonard, G., Pike, G. B., \& Paus, T. (2011). Development of the action observation network during early adolescence: A longitudinal study. Social Cognitive and Affective Neuroscience, 1, 1-17.

Spear, L. P. (2000). The adolescent brain and age-related behavioral manifestations. Neuroscience and Biobehavioral Reviews, 24, 417-463.

Steinberg, L. (2008). A social neuroscience perspective on adolescent risk-taking. Developmental Reviews, 28, 78-101.

Tahmasebi, A. M., Artiges, E., Banaschewski, T., Barker, G. J., Bruehl, R., Büchel, C., et al. (2001). Creating probabilistic maps of the face network in the adolescent brain: A multicentre functional MRI study. Human Brain Mapping, Epub ahead of print. doi: 10.1002/hbm.21261.

Toro, R., Fox, P., \& Paus, T. (2008). Function coactivation map of the human brain. Cerebral Cortex, 18, 2553-2559.

Uddin, L. Q., Iacoboni, M., Lange, C., \& Keenan, P. (2007). The self and social cognition: The role of cortical midline structures and mirror neurons. Trends in Cognitive Science, 11, 153-157.

Van Overwalle, F. (2010). Social cognition and the brain: A meta-analysis. Human Brain Mapping, 30, 829-858.

Van Overwalle, F., \& Baetens, K. (2009). Understanding others' actions and goals by mirror and mentalising systems: A meta-analysis. Neuroimage, 48, 564-584. 